\title{
Reply to: Yes, and no - evidence on lung ultrasound digested
}

\section{Paolo Tomà ${ }^{1}$}

Received: 20 March 2020 / Revised: 20 March 2020 / Accepted: 16 April 2020 / Published online: 12 May 2020

(C) Springer-Verlag GmbH Germany, part of Springer Nature 2020

\section{Dear Editors,}

I thank you for your stimulating and valuable comment [1]. Please note that it is not my intention to turn the journal into a monthly philosophy magazine. In fact, I believe that the distinction between deductive and inductive methods is undoubtedly a cornerstone of the philosophy of science.

Nevertheless, the influence of social and cultural values on the very structure of knowledge is indisputable. Scientific knowledge is the result not only of our interactions, especially the experimental ones, with the material world around us, but also of interactions between individuals and society.

Ethical, social and political values come into play — and this is the most debated aspect - in the key moments of research, in the collection of evidence and in the recognition of a certain hypothesis as an adequate response to a specific scientific question in the light of the acquired evidence.

We have to focus not only on institutional science, laboratories and research centers, but also on applications, languages and the means of communication through which science is disseminated. This is the reason why my paper starts with historical assumptions [2]. It does not aim to enhance the past to prevent the future, but to understand the dynamics that led to the evolution of ultrasound in the study of the lung by a group of professionals who, coming from different cultures, albeit similar, have gone beyond fossilized approach concepts.

On the one hand, radiologists were substantially convinced that ultrasound was an outdated technique with limited evolutionary potential. Additionally, from a scientific point of view, they were wholly oriented toward the most rewarding sophisticated technology, such as magnetic resonance imaging.

Non-radiologists, on the other side, thanks to portable machines, discovered a means that could be a direct support for particular emergencies to the clinical approach. Two different positions, with sometimes naïve debates, over time have become a source of collaboration and convergence of opinions from both scientific and practical points of view. In my hospital, young radiologists have learned to integrate chest radiography with ultrasound. Clinicians rationalized their ultrasound approach by including limits, resulting from an enthusiastic initial immature monomodal malpractice. The same effort has been made by radiologists trained in corporate conservatism. It is a dynamic and collective process, the expression of a scientific community that recognizes itself in a core of common standards and establishes places for sharing and checking results. A plurality of perspectives contributes positively to the reliability of scientific knowledge, whose rationality is assured by the comparison between different positions, guaranteeing a role for all relevant points of view. In an era in which the diffusion of information - including that relating to discoveries, advances and failures of science - is much more widespread and faster, the relationship between science and public opinion is also subject to rapid changes. Theoretical reflection is called first to commit to safeguarding genuinely scientific contents - distinguishing them from nonscientific ones which, however, claim to be such.

\section{References}

1. Olsen ØE (2020) Yes, and no - evidence on lung ultrasound digested. Pediatr Radiol 50:305

2. Tomà $\mathrm{P}$ (2020) Lung ultrasound in pediatric radiology - cons. Pediatr Radiol 50:314-320

Publisher's note Springer Nature remains neutral with regard to jurisdictional claims in published maps and institutional affiliations.
Paolo Tomà

paolo.toma@opbg.net

1 Ospedale pediatrico Bambino Gesù, Piazza S.Onofrio 4, 00165 Rome, Italy 